\title{
PreSSUB II: The prehospital stroke study at the Universitair Ziekenhuis Brussel II
}

\begin{abstract}
Alexis Valenzuela Espinoza ${ }^{1,2}$, Robbert-Jan Van Hooff ${ }^{1,3}$, Ann De Smedt ${ }^{1,3}$, Maarten Moens ${ }^{1,4}$, Laetitia Yperzeele ${ }^{1,3,5}$, Koenraad Nieboer ${ }^{6}$, Ives Hubloue ${ }^{7}$, Jacques De Keyser ${ }^{1,3,8}$, Alain Dupont ${ }^{9}$, Liesbet De Wit ${ }^{10}$, Koen Putman ${ }^{2,10}$, Raf Brouns ${ }^{1,3}$

${ }^{1}$ Center for Neurosciences (C4N), ${ }^{2}$ Interuniversity Center for Health Economics Research (I-CHER), Vrije Universiteit Brussel (VUB), Laarbeeklaan 103, ${ }^{3}$ Departments of Neurology, ${ }^{4}$ Neurosurgery and ${ }^{6}$ Radiology, Universitair Ziekenhuis Brussel, ${ }^{9}$ Research Group Clinical Pharmacology and Clinical Pharmacy (KFAR), ${ }^{10}$ Public Health, Vrije Universiteit Brussel (VUB), Laarbeeklaan 101, 1090 Brussels, ${ }^{5}$ Department of Neurology, Universitair Ziekenhuis Antwerpen, Wilrijkstraat 10, 2650 Edegem, 'Department of Emergency Medicine, Universitair Ziekenhuis Brussel, Laarbeeklaan 101, 1090 Brussels, Belgium and Research Group on Emergency and Disaster Medicine (ReGEDiM), Vrije Universiteit Brussel (VUB), Laarbeeklaan 103, 1090 Brussels, Belgium, ${ }^{8}$ Department of Neurology, University Medical Center Groningen, University of Groningen, Hanzeplein 1, 9713 GZ Groningen, Netherlands
\end{abstract}

\begin{abstract}
Rationale: Stroke is a time-critical medical emergency requiring specialized treatment. Prehospital delay contributes significantly to delayed or missed treatment opportunities. In-ambulance telemedicine can bring stroke expertise to the prehospital arena and facilitate this complex diagnostic and therapeutic process. Aims: This study evaluates the efficacy, safety, feasibility, reliability and cost-effectiveness of in-ambulance telemedicine for patients with suspicion of acute stroke. We hypothesize that this approach will reduce the delay to in-hospital treatment by streamlining the diagnostic process and that prehospital stroke care will be improved by expert stroke support via telemedicine during the ambulance transportation. Design: PreSSUB II is an interventional, prospective, randomized, open-blinded, end-point, single-center trial comparing standard emergency care by the Paramedic Intervention Team of the Universitair Ziekenhuis Brussel (control) with standard emergency care complemented with in-ambulance teleconsultation service by stroke experts (PreSSUB). Study Outcomes: The primary efficacy endpoint is the call-to-brain imaging time. Secondary endpoints for the efficacy analysis include the prevalence of medical events diagnosed and corrected during in-ambulance teleconsultation, the proportion of patients with ischemic stroke receiving recanalization therapy, the assessment of disability, functional status, quality of life and overall well-being. Mortality at 90 days after stroke is the primary safety endpoint. Secondary safety analysis will involve the registration of any adverse event. Other analyses include assessment of feasibility and reliability and a health economic evaluation.
\end{abstract}

Key words: Emergency medicine, prehospital, stroke, telemedicine

\section{INTRODUCTION}

Stroke is a time-critical medical emergency requiring specialized treatment. The medical and economic burden of stroke can be mitigated by therapies focusing on rapid reperfusion of the occluded blood vessel and protection of viable nervous tissue in the penumbra (neuroprotection).$^{[1]}$
Pursuant to technological developments and in line with the American Stroke Association and the European Stroke Organization guidelines, the implementation of telemedicine for optimizing in-hospital stroke care (telestroke) is currently applied in several countries. ${ }^{[2-8]}$ Telemedicine is defined as the use of information and communication technology to provide 
healthcare services to individuals who are at a distance from a specialized healthcare provider ${ }^{[0]}$ and has shown to be a valid, accurate and reliable tool for improving in-hospital stroke care. ${ }^{[8,10,11]}$

Research on prehospital telemedicine for acute stroke care is a promising concept ${ }^{[12]}$ and is recommended by the American Stroke Association, ${ }^{[13]}$ but exploration of realtime in-ambulance telestroke has been limited to studies in healthy volunteers ${ }^{[14-16]}$ and two feasibility studies in patients during emergency missions. ${ }^{[17,18]}$ A pilot study evaluating the safety and the technical feasibility of inambulance telestroke is currently ongoing (Pre-hospital Stroke Study at the Universitair ziekenhuis Brussel I, PreSSUB I).

PreSSUB II will evaluate the efficacy, safety, feasibility, reliability and cost-effectiveness of in-ambulance telemedicine for patients with suspicion of acute stroke. We hypothesize that in-ambulance telestroke will reduce the delay to in-hospital treatment by streamlining the diagnostic process and that pre-hospital stroke care will be improved by expert stroke support via telemedicine during the ambulance transportation.

\section{MATERIALS AND METHODS}

\section{Design}

The PreSSUB II is an interventional, prospective, randomized, open-blinded, end-point, single-center trial comparing standard emergency care by the Paramedic Intervention Team (PIT) of the Universitair Ziekenhuis Brussel (UZB) (control) with this standard emergency care complemented with in-ambulance teleconsultation service by stroke experts (PreSSUB).

\section{Randomization}

Randomization is obtained on a weekly basis by block randomization using two alternative blocks of 4 weeks, i.e. "PreSSUB - control - PreSSUB - control" or "control - PreSSUB - control — PreSSUB." All patients will receive in-hospital care and follow-up according to current standing operating procedures of the UZB [Figure 1]. The outcome parameters will be assessed by raters who are blinded for the treatment allocation.

\section{Patient population}

The PIT of the UZB serves the area of the northwest of Brussels, consisting of about half a million inhabitants. The catchment area is identical during the control weeks and the PreSSUB weeks. The PIT is available $24 \mathrm{~h}$ a day, 7 days a week as part of routine clinical practice. Additionally, round-the-clock availability of the prehospital telemedicine service will be provided during the PreSSUB weeks.

We will include all patients who are transported to the UZB by the PIT of the UZB for symptoms suggestive of a stroke with onset less than $12 \mathrm{~h}$ or unknown time of onset, unless patients are younger than 18 years. A clinical suspicion of stroke will be based on any of the symptoms mentioned in the Belgian Manual for Medical Regulation of Prehospital Care, ${ }^{[19]}$ including hemiparesis, facial asymmetry, speech disturbance, sudden severe headache or confusion. Patients in whom in-ambulance telemedicine consultation would delay any diagnostic or therapeutic intervention are excluded.

Given the time-critical nature of acute stroke care, and in line with the procedure applied in the FACT

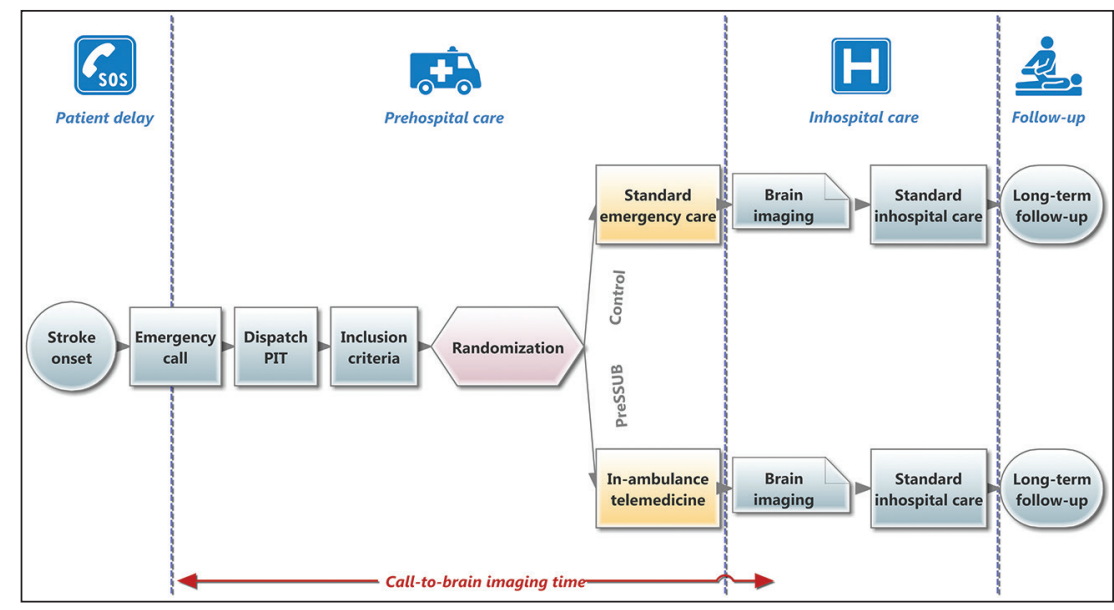

Figure 1: The study design of the prehospital stroke study at the Universitair ziekenhuis Brussel II

Source: Based on the information provided during the emergency call, the emergency call-taker will dispatch the Paramedic Intervention Team (PIT) of the Universitair Ziekenhuis Brussel to the patient. Eligible patients are allocated on a weekly basis to either standard care (control) or standard care supplemented with in-ambulance telemedicine (PreSSUB). All patients receive standard in-hospital care and long-term follow-up. The call-to-brain imaging time is registered as the primary efficacy endpoint 
study, ${ }^{[18]}$ informed consent will be requested based on the "opt-out sampling" principle. This implies that consent will be requested from the patients or their legal representatives after the ambulance transportation, when the patient has been stabilized in the hospital. The study protocol and the consent process were approved by the ethics committee of the UZB (EUDRACT 2014-003463-38). The study was registered at clinicaltrials.gov (NCT02270541).

\section{Control}

During control weeks, patients receive standard emergency care by the PIT of the UZB according to current clinical practice based on the standing operating procedures and the Belgian legislation. ${ }^{[20]}$ Upon emergency call by a patient or bystander, the emergency call-taker dispatches the PIT to the patient's location in accordance with the Belgian Manual for Medical Regulation for Prehospital Care. ${ }^{[19]}$ The PIT nurse may at all times request telephone support by a dedicated in-hospital emergency physician or on-site support by a medical emergency team consisting of an emergency physician and an emergency nurse.

\section{Intervention}

The in-ambulance teleconsultation service is provided 24/7 during the PreSSUB weeks. After notification by the PIT-nurse, the teleconsultant accesses the secured web-based telemedicine platform using a dedicated laptop and WiFi or $3 \mathrm{G} / 4 \mathrm{G}$ connection. Teleconsultations are performed by the National Institutes of Health Stroke Scale-certified stroke experts with experience in telestroke and the Unassisted Telestroke Scale (UTSS, Table 1). ${ }^{[15,18,21]}$ Patients are examined in the ambulance via bidirectional audio - video communication using the PreSSUB device [Figure 2] while secured on a stretcher in the supine position, in line with safety regulations. The head of the stretcher is positioned according to patient's condition (preferably at 30 degrees). The ambulance is normally lit (standard lightning) and the sirens are activated as required. All patients will receive care by the PIT-nurses according to the current clinical practice based on standing operating procedures. ${ }^{[20]}$ After the patient is brought aboard the ambulance, the vital functions and glycemia are registered using the PIT's standard equipment and are orally communicated to the teleconsultant or observed on the

\section{Table 1: The Unassisted TeleStroke Scale (UTSS)}

\begin{tabular}{|c|c|c|}
\hline Item & Instruction & Scale definition \\
\hline Consciousness & "How are you?" & $\begin{array}{l}0 \text { Verbal or motor response to speech } \\
1 \text { No verbal or motor response to speech }\end{array}$ \\
\hline Orientation & "Please tell me what month we are in" & $\begin{array}{l}0 \text { Correct } \\
1 \text { Incorrect }\end{array}$ \\
\hline Eye position at rest & "Please look straight ahead" & $\begin{array}{l}0 \text { Normal eye position } \\
1 \text { Conjugate deviation, divergent position or } \\
\text { involuntary movement }\end{array}$ \\
\hline Voluntary eye movement & "Please look left and right without moving your head" & $\begin{array}{l}0 \text { Normal } \\
1 \text { Limited horizontal range of one or both eyes }\end{array}$ \\
\hline Head position at rest & Observe the spontaneous head position & $\begin{array}{l}0 \text { Midline position } \\
1 \text { Deviation to one side }\end{array}$ \\
\hline Motor face & $\begin{array}{l}\text { "Please close your eyes tightly" } \\
\text { Observe motor activity of the entire facial musculature }\end{array}$ & $\begin{array}{l}0 \text { Normal, symmetrical facial movement } \\
1 \text { Asymmetrical or absent facial movement }\end{array}$ \\
\hline Motor left arm & $\begin{array}{l}\text { "Please hold your left arm extended at } 45^{\circ} \text { for } 5 \\
\text { seconds" }\end{array}$ & $\begin{array}{l}0 \text { Normal } \\
1 \text { Arm cannot be held at } 45^{\circ} \text { for } 5 \text { seconds }\end{array}$ \\
\hline Motor right arm & $\begin{array}{l}\text { "Please hold your right arm extended at } 45^{\circ} \text { for } 5 \\
\text { seconds" }\end{array}$ & $\begin{array}{l}0 \text { Normal } \\
1 \text { Arm cannot be held at } 45^{\circ} \text { for } 5 \text { seconds }\end{array}$ \\
\hline Motor left hand & $\begin{array}{l}\text { "Please spread the fingers of your left hand as wide } \\
\text { apart as you can" }\end{array}$ & $\begin{array}{l}0 \text { Normal } \\
1 \text { The fingers cannot be spread widely }\end{array}$ \\
\hline Motor right hand & $\begin{array}{l}\text { "Please spread the fingers of your right hand as wide } \\
\text { apart as you can" }\end{array}$ & $\begin{array}{l}0 \text { Normal } \\
1 \text { The fingers cannot be spread widely }\end{array}$ \\
\hline Motor left foot & "Please pull the toes of your left foot towards you" & $\begin{array}{l}0 \text { Normal dorsiflexion } \\
1 \text { Incomplete or absent dorsiflexion }\end{array}$ \\
\hline Motor right foot & "Please pull the toes of your right foot towards you" & $\begin{array}{l}0 \text { Normal dorsiflexion } \\
1 \text { Incomplete or absent dorsiflexion }\end{array}$ \\
\hline Naming & $\begin{array}{l}\text { "How do you call a horse with black and white } \\
\text { stripes?" }\end{array}$ & $\begin{array}{l}0 \text { Correct } \\
1 \text { Incorrect }\end{array}$ \\
\hline Repetition & $\begin{array}{l}\text { "Please repeat after me. He forgot to store his bike } \\
\text { indoors" }\end{array}$ & $\begin{array}{l}0 \text { Correct repetition } \\
1 \text { Incorrect repetition }\end{array}$ \\
\hline Articulation & $\begin{array}{l}\text { Assess the clarity of articulation throughout the entire } \\
\text { examination }\end{array}$ & $\begin{array}{l}0 \text { Normal articulation } \\
1 \text { Dysarthria }\end{array}$ \\
\hline $\begin{array}{l}\text { Spatial attention and left/ } \\
\text { right orientation }\end{array}$ & $\begin{array}{l}\text { Assess spatial attention and left/right orientation } \\
\text { throughout the entire examination }\end{array}$ & $\begin{array}{l}0 \text { Normal } \\
1 \text { Hemi-inattention or left/right confusion }\end{array}$ \\
\hline
\end{tabular}

Assessment of the 16 items is facilitated and standardized by a software tool, presenting the instructions to the rater in the language preferred by the patient (Dutch, French or English). The text between quotation marks should be read literally to the patient 
monitor screen by the teleconsultant. Patient identification is obtained using the patient's e-ID card, if available. After insertion of the e-ID card in the telemedicine device, the patient's name and date of birth are automatically sent to the web-based telemedicine platform [Figure 3]. The teleconsultant can support the PIT-nurse for obtaining and maintaining homeostasis during ambulance transportation. If necessary, the teleconsultant can assist the PIT-nurse for optimal application of the PIT's standard operating procedures regarding airway protection, blood oxygen saturation, arterial blood pressure, heart rate, cardiac arrhythmia, decreased level of consciousness, dysglycemia and other supportive measures (e.g., anti-emetics, analgetics). Whenever possible and without interfering with the PIT's routine practice, the teleconsultant assesses the patient's neurological status using the Glasgow Coma Scale (GCS) and the UTSS. The teleconsultant attempts to take

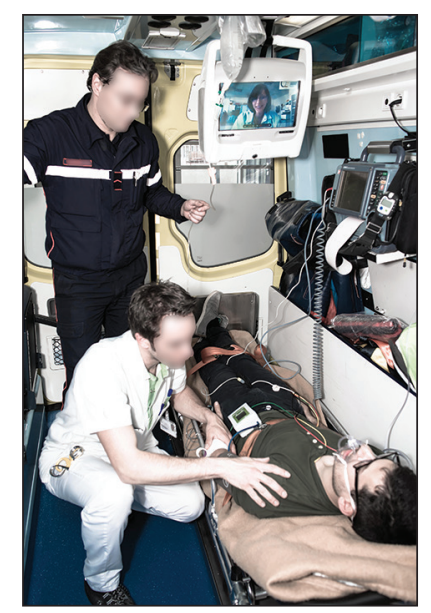

Figure 2: The PreSSUB system in the ambulance

Source: Bidirectional audiovisual communication between the patient and the teleconsultant is possible via the telemedicine device that is mounted to the ceiling of the ambulance a medical history from the patient focusing on the time of symptom onset, past medical history and medication use. The teleconsultant may formulate a preliminary diagnosis and attempts can be made to identify potential candidates for recanalization therapy. Additionally, information can be gathered regarding body weight, prehospital interventions, contact information of the family or prestroke functional state. The teleconsultant may activate the "Code Stroke" procedure (prioritized in-hospital care for patients with suspicion of acute stroke) if a potential candidate for recanalization therapy is identified. For this, he activates an automated message from the telemedicine platform to notify the in-hospital stroke team (consisting of an emergency physician, neurologist and neuroradiologist). All acquired data are communicated to the in-hospital team via personal, password-protected encrypted access to the teleconsultation report on the telemedicine platform. The report can be accessed by emergency physicians, radiologists and neurologists involved in the patient's care using any computer, tablet or smartphone with access to the Internet. Upon arrival at the hospital, patient care continues according to current clinical practice. To evaluate and scrutinize the teleconsultation, screen recordings from the teleconsultant's computer are made. These will be used for intra- and interrater evaluation of the various clinical scales and the care process. The bandwidth and technical issues of the mobile connection during the teleconsultation will be measured at the teleconsultant's side using bandwidth monitoring software.

\section{Outcomes}

Efficacy analysis

The primary endpoint is the call-to-brain imaging time, referring to the interval between the emergency call to the emergency dispatch center and the in-hospital brain

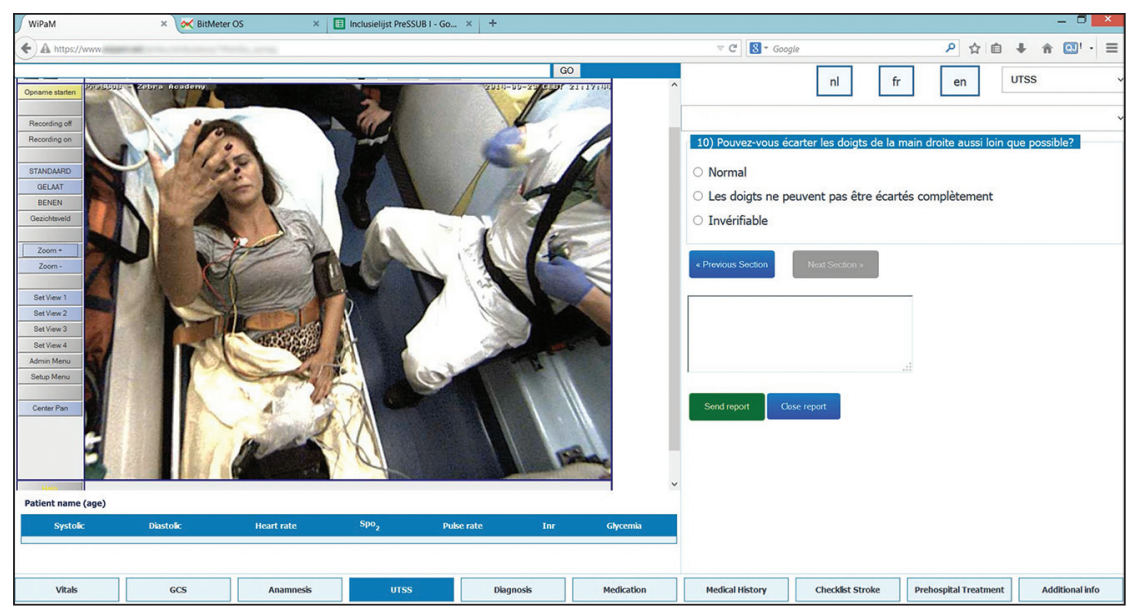

Figure 3: The PreSSUB telemedicine platform

Source: Screen print of the PreSSUB telemedicine platform showing the clinical support system for stroke diagnosis. The left side of the screen shows video input from the ambulance (i.e., the patient spreading the fingers of her right hand). The right side shows the submenu of the support system that is activated (i.e., item 10 of the Unassisted TeleStroke Scale in the language preferred by the patient). Navigation between the various submenus is facilitated via touch screen activation of the buttons at the bottom 
imaging, either by computed tomography or magnetic resonance imaging. An average time gain of $20 \mathrm{~min}$ in the diagnostic and therapeutic process is regarded as clinically relevant because this is likely to translate in improved long-term stroke outcome at the population level. ${ }^{[12]}$ The following secondary efficacy endpoints will be studied:

1. The prevalence of medical events diagnosed and corrected according to the current standing operating procedures for prehospital care at the $\mathrm{UZB}^{[20]}$ during the in-ambulance teleconsultation,

2. The proportion of patients with ischemic stroke receiving recanalization therapy,

3. The assessment of the disability, the functional status, the quality of life and overall well-being using the modified Rankin Scale (mRS), ${ }^{[22]}$ Barthel Index, ${ }^{[23]}$ Euroquol (EQ-5D) ${ }^{[24]}$ and WHO-Five Well-Being Index, ${ }^{[25]}$ respectively, at 30, 90, 180 and 365 days after stroke.

\section{Safety analysis}

Mortality at 90 days after stroke will be the primary safety endpoint. Secondary safety analysis will involve the registration of any adverse event and will look particularly at known complications of delayed stroke care and thrombolytic therapy (in-hospital mortality, symptomatic intracranial hemorrhage, systemic bleeding after thrombolytic therapy requiring transfusion or inappropriate use of recanalization therapy, as defined by review of the patient files and video recordings by a panel of independent stroke experts).

Feasibility analysis

The endpoints for feasibility analysis include:

1. The proportion of successful in-ambulance telemedicine consultations,

2. The proportion of successful prehospital "Code Stroke" activations,

3. The prevalence of technical issues with regard to wireless connectivity, audio quality, video quality, webbased telemedicine platform,

4. Questionnaire-based acceptance of all stakeholders,

5. Prehospital time consumption (e.g., call-to-admission time, duration of the teleconsultation) and

6. Technical observations regarding the bandwidth of the mobile connection.

Reliability analysis

The concurrent validity of the prehospital telemedicine diagnosis and assessment of clinical scales (GCS and UTSS) will be evaluated by correlation with the final inhospital diagnosis and clinical scales. The reliability of the prehospital telemedicine diagnosis and assessment of clinical scales (GCS and UTSS) will be evaluated by the interrater variability, the intrarater variability and the rater agreement using the video recordings of the teleconsultations. The predictive validity of the UTSS obtained via in-ambulance telemedicine will be assessed by correlation with the mRS score and mortality at 90 days. The accuracy of the UTSS to differentiate between largevessel and small-vessel stroke will be assessed by correlation with the Oxfordshire Community Stroke Project (OCSP) classification and with the vessel status obtained in-hospital.

Health economic evaluation

A cost-utility analysis with societal perspective will be performed comparing the teleconsultation group with the standard care group. Resource utilization in both groups will be derived from claims' data during hospitalization and self-reporting for the period post discharge. Unit costs will be derived from national registries. Utilities will be measured by the EQ-5D surveys at 1 year post stroke. The health economic evaluation will be performed by decision - analytical modeling. A decision tree will be developed and populated with trial data wherever possible, which will model the short-term consequences (for the duration of hospital stay). Additionally, a Markov model will be used to model the outcomes over a long-term horizon. The latter will be based on national data ${ }^{[26]}$ and findings from the CERISE study ${ }^{[27-29]}$ and completed with information from the international peer-reviewed literature. A probabilistic sensitivity analysis, in which the crucial variables will be varied, will be performed to account for the uncertainties. Incremental cost-effectiveness ratios will be calculated and evaluated on willingness-to-pay thresholds.

\section{Data Safety Monitoring Board (DSMB)}

An independent DSMB has reviewed and approved the trial protocol and will monitor the conduction of the trial. Interim analyses of adverse events will be performed after enrolment of 46 and 92 patients. The DSMB will continuously monitor the rate of serious adverse events and suspected unexpected adverse reactions. The DSMB may at any time recommend an amendment to the clinical trial protocol or termination of the trial in case of safety concerns.

\section{Sample size}

The sample size calculation is based on the primary efficacy endpoint (i.e., call-to-brain imaging time). Thanks to the facilitation of the stroke diagnosis process by in-ambulance telemedicine, we expect the call-to-brain imaging time on average to be 20 min less in the PreSSUB group compared with the control group. Accurate national information regarding call-to-brain imaging times for patients arriving at emergency departments by ambulances is not available. The best estimate for the average call-to-brain imaging time in UZB is $110 \mathrm{~min}$ (standard deviation, $40 \mathrm{~min}$ ). In order to demonstrate the expected reduction in call-to- 
brain imaging time (i.e., to reject the null hypothesis of an identical call-to-brain imaging time in both groups) with a type- 1 error $\alpha=0.05$ (two-tailed), a power of $80 \%$ $(\beta=20 \%)$ is achieved with a sample size of 128 patients ( $n=64$ per treatment group). Accounting for possible treatment failures, protocol violations and drop-outs, we plan to enroll 140 patients ( $n=70$ per treatment group).

\section{Statistical plan}

All analyses will be conducted according to the intentionto-treat principle and the per protocol principle. All efforts will be made to minimize the amount of missing data. Sensitivity analyses based on different hypotheses about the missingness pattern of the primary outcome will be performed to test for the robustness of the primary analysis.

Following a descriptive analysis, the distribution of outcomes between patient groups will be compared using the Mann-Whitney $\mathrm{U}$ test, the $\chi^{2}$ test or the Fisher exact test. Multivariate regression analysis will be performed and shift analysis of the mRS score will be assessed by the van Elteren Cochran-Mantel-Hanszel test. Survival analysis using the Kaplan-Meier method and the Cox proportional hazards multivariate analysis will be performed. The interrater variability and the intrarater rater variability will be evaluated using the Spearman's $\rho$ correlation, the $\chi^{2}$ test or the Fisher exact test. The rater agreement will be tested using the intraclass correlation coefficient for single measures and weighted c statistics. The concurrent validity of the clinical scales will be assessed by the Spearman's $\rho$ correlation. To assess the UTSS' predictive validity for outcome and its accuracy to differentiate between large-vessel and smallvessel stroke, we will assess the Spearman's $\rho$ correlation with the mRS score and the correlation with the OCSP classification and with the vessel status obtained inhospital. Receiver operating curves, $\boldsymbol{\chi}$-statistics, optimal thresholds, likelihood ratios and accuracy measures will be calculated. Linear or generalized linear models will be used to analyze determinants for health care utilization and costs. A similar approach will be used to compare the level and evolution of quality of life and well-being between the intervention and the control group.

\section{Study organization and funding}

The study will be conducted by the PreSSUB-consortium consisting of the Departments of Neurology, Emergency Medicine, Neurosurgery and Radiology at the UZB, the Center for Neurosciences, the Interuniversity Center for Health Economics Research, the Research Group Clinical Pharmacology and Clinical Pharmacy and the Research Group on Emergency and Disaster Medicine. The consortium collaborates closely with the Department of Electronics and Informatics and the interdisciplinary Research Group on Law Science Technology and Society of the Vrije Universiteit Brussel, the OPERA department and the Department of Neurology of the Université Libre de Bruxelles, and the Neurology department of the Universite Catholique de Louvain. The study receives funding from the Brussels Institute for Research and Innovation, the Research Foundation - Flanders, the Strategic Research Project Growth Fund and the Industrial Research Fund of the Vrije Universiteit Brussel, the Scientific Fund Willy Gepts of the Universitair Ziekenhuis Brussel and the Caring Entrepreneurship Fund of the King Baudouin Foundation. The sponsors are not involved in the study design and have no influence on the analysis, interpretation or publication of the data.

\section{SUMMARY}

We developed a system for in-ambulance telemedicine aiming to improve prehospital stroke care. This system consists of a telemedicine device in the ambulance, a telemedicine platform supporting the teleconsultant in the diagnostic and therapeutic process and a system for notification and reporting to the in-hospital team. The efficacy, safety, feasibility, reliability and costeffectiveness of this approach will be evaluated in the PreSSUB II.

\section{ACKNOWLEDGMENTS}

The authors are grateful to Geert Vanhooren, Caroline Leigh Watkins and Ronald Buyl for their contribution as members of the Data Monitoring Safety Board, and to Gerben DeJong and Vincent Thijs for their contribution as members of the Scientific Advisory Board. The authors also thank Jean-Marie Ledeghen, Maximiliaan Wyns and the SIAMU (Service d'Incendie et d'Aide Médicale Urgente de la Région de Bruxelles-Capitale) for their support and collaboration.

\section{REFERENCES}

1. Manning NW, Campbell BC, Oxley TJ, Chapot R. Acute ischemic stroke: Time, penumbra, and reperfusion. Stroke 2014;45:640-4.

2. Hess DC, Audebert HJ. The history and future of telestroke. Nat Rev Neurol 2013;9:340-50.

3. Rubin MN, Wellik KE, Channer DD, Demaerschalk BM. A systematic review of telestroke. Postgrad Med 2013;125:45-50.

4. LaMonte MP, Cullen J, Gagliano DM, Gunawardane R, Hu P, Mackenzie C, et al. TeleBAT: Mobile telemedicine for the Brain Attack Team. J Stroke Cerebrovasc Dis 2000;9:128-35.

5. LaMonte MP, Xiao Y, Hu PF, Gagliano DM, Bahouth MN, Gunawardane RD, et al. Shortening time to stroke treatment using ambulance telemedicine: TeleBAT. J Stroke Cerebrovasc Dis 2004;13:148-54.

6. Audebert HJ, Tietz V, Boy S, Pilz P, Haberl RL, Schenkel J. Acceptance of telemedicine for acute stroke care. The German project TEMPiS. Nervenarzt 2009;80:184-9.

7. Tatlisumak T, Soinila S, Kaste M. Telestroke networking offers multiple benefits beyond thrombolysis. Cerebrovasc Dis 2009;27 Suppl 4:21-7. 
8. Sairanen $\mathrm{T}$, Soinila $\mathrm{S}$, Nikkanen M, Rantanen $\mathrm{K}$, Mustanoja $\mathrm{S}$, Farkkila M, et al. Two years of Finnish Telestroke: Thrombolysis at spokes equal to that at the hub. Neurology 2011;76:1145-52.

9. Roine R, Ohinmaa A, Hailey D. Assessing telemedicine: A systematic review of the literature. CMAJ 2001;165:765-71.

10. Audebert HJ, Kukla C, Vatankhah B, Gotzler B, Schenkel J, Hofer S, et al. Comparison of tissue plasminogen activator administration management between Telestroke Network hospitals and academic stroke centers: The Telemedical Pilot Project for Integrative Stroke Care in Bavaria/Germany. Stroke 2006;37:1822-7.

11. Vatankhah B, Schenkel J, Furst A, Haberl RL, Audebert HJ. Telemedically provided stroke expertise beyond normal working hours. The Telemedical Project for Integrative Stroke Care. Cerebrovasc Dis 2008;25:332-7.

12. Yperzeele L, Van Hooff RJ, de Smedt A, Valenzuela Espinoza A, Van de Casseye R, Hubloue I, et al. Prehospital stroke care: Limitations of current interventions and focus on new developments. Cerebrovasc Dis 2014;38:1-9.

13. Schwamm LH, Audebert HJ, Amarenco P, Chumbler NR, Frankel MR, George MG, et al. Recommendations for the implementation of telemedicine within stroke systems of care: A policy statement from the American Heart Association. Stroke 2009;40:2635-60.

14. Liman TG, Winter B, Waldschmidt C, Zerbe N, Hufnagl P, Audebert $\mathrm{HJ}$, et al. Telestroke ambulances in prehospital stroke management: Concept and pilot feasibility study. Stroke 2012;43:2086-90.

15. Van Hooff RJ, Cambron M, Van Dyck R, De Smedt A, Moens M, Valenzuela Espinoza A, et al. Prehospital unassisted assessment of stroke severity using telemedicine: A feasibility study. Stroke 2013;44:2907-9.

16. Wu TC, Nguyen C, Ankrom C, Yang J, Persse D, Vahidy F, et al. Prehospital utility of rapid stroke evaluation using in-ambulance telemedicine: A pilot feasibility study. Stroke 2014;45:2342-7.

17. Bergrath S, Reich A, Rossaint R, Rortgen D, Gerber J, Fischermann H, et al. Feasibility of prehospital teleconsultation in acute stroke-a pilot study in clinical routine. PLoS One 2012;7:e36796.

18. Yperzeele L, Van Hooff RJ, De Smedt A, Valenzuela Espinoza A, Van Dyck R, Van de Casseye R, et al. Feasibility of AmbulanCe-based Telemedicine (FACT) study: Safety, feasibility and reliability of third generation in-ambulance telemedicine. PLoS One 2014;9:e110043.
19. Federal Public Service Health Food chain Safety and Environment. [Belgian manual for medical regulation]. 2008

20. Bronselaer K, Todorov P, Stamatakis L. [PIT experiment 2007-2008 Final report]. Federal Public Service Health, Food chain safety and Environment. 2009

21. Van Hooff RJ, De Smedt A, De Raedt S, Moens M, Marien P, Paquier P, et al. Unassisted assessment of stroke severity using telemedicine. Stroke 2013;44:1249-55.

22. van Swieten JC, Koudstaal PJ, Visser MC, Schouten HJ, van Gijn J. Interobserver agreement for the assessment of handicap in stroke patients. Stroke 1988;19:604-7.

23. Mahoney FI, Barthel DW. Functional Evaluation: The Barthel Index. Md State Med J 1965;14:61-5.

24. EuroQol Group. EuroQol--a new facility for the measurement of health-related quality of life. Health Policy 1990;16:199-208.

25. Bonsignore M, Barkow $K$, Jessen $F$, Heun R. Validity of the five-item WHO Well-Being Index (WHO-5) in an elderly population. Eur Arch Psychiatry Clin Neurosci 2001;251 Suppl 2:II27-31.

26. Kiekens C, Van Rie K, Leys M, Cleemput I, Smet M, Kesteloot K, et al. [Organisation and financing of musculo skeletal and neuro rehabilitation in Belgium]. 2007.

27. De Wit L, Putman K, Devos H, Brinkmann N, Dejaeger E, De Weerdt W, et al. Five-year mortality and related prognostic factors after inpatient stroke rehabilitation: A European multi-centre study. J Rehabil Med 2012;44:547-52.

28. Putman K, De Wit L, Schupp W, Baert I, Brinkmann N, Dejaeger E, et al. Variations in follow-up services after inpatient stroke rehabilitation: A multicentre study. J Rehabil Med 2009;41:646-53.

29. De Wit L, Putman K, Schuback B, Komarek A, Angst F, Baert I, et al. Motor and functional recovery after stroke: A comparison of 4 European rehabilitation centers. Stroke 2007;38:2101-7.

How to cite this article: Espinoza AV, et al. PreSSUB II: The prehospital stroke study at the Universitair Ziekenhuis Brussel II. J TransI Intern Med 2015;3:57-63

Source of Support: NIL, Conflict of Interest: NIL 